\section{Urine diacetylated spermine might be a nonspecific tumor marker in canine neoplasia}

\author{
Isaka Mitsuhiro, 1 Ito Noriko, 1 \\ Kuwahara Takeshi, 1 Takahasi Yosuke, ${ }^{1}$ \\ Onoue Midori, ${ }^{1}$ Arase Yurie, ${ }^{1}$ \\ Namba Shinichi,1 Ryuichi Sekizawa² \\ 1Marble Veterinary Medical Center, \\ Ishikawa, Fujisawa, Kanagawa; \\ 2Metaboscreen Co., Ltd. Venture Business \\ Laboratory 211, Yokohama National \\ University, Yokohama, Kanagawa, Japan
}

\section{Abstract}

Diacetylated spermine, a polyamine, is clinically used as a tumor marker in human medicine. The purpose of this study was to evaluate the usefulness of diacetylated spermine as a nonspecific tumor marker in dogs. One hundred six dogs were randomly selected and divided into 4 groups (cancer-bearing, $\mathrm{n}=23$; control, $\mathrm{n}=44$; young, $\mathrm{n}=24$; inflammatory, $\mathrm{n}=15$ ). Urine samples were collected to measure diacetylated spermine and creatinine levels. The diacetylated spermine concentration was determined by enzyme-linked immunoassay, standardized by creatinine concentration. The urine diacetylated spermine concentration was markedly significant elevated in cancerbearing dogs, as compared to that in control dogs $(\mathrm{P}=0.0081)$. Whilst the urine diacetylated spermine level in cancer-bearing group was not markedly different from that in the young group, there was significant different between levels in the control and young groups $(\mathrm{P}=0.019)$. No significant difference was observed between the inflammatory and cancer-bearing groups. It is suggested that urine diacetylated spermineis a useful tumor marker in dogs over 2 years of age.

\section{Introduction}

Presently, the diagnosis and examination of canine cancer patients involves procedures such as histopathological examination, ultrasonography, computed tomography (CT), and magnetic resonance imaging (MRI). However, expense and/or systemic anesthesia for diagnostic imaging are unfavorable factors. Recently, diagnosis using tumor markers has become topical issue in veterinary medicine. Early detection of cancer is possible, if tumor markers are clinically available; however, there are limited reports about the usefulness of tumor markers in dogs. 1,2 Some researchers in the veterinary field have studied tumor markers used in human medicine.

In 1971, the excess urinary excretion of polyamines was reported in human cancer patients. ${ }^{3}$ Polyamines are classified according to various structures, ${ }^{4}$ and diacetylated spermine, a modified polyamine, is reported to have excellent characteristics as a tumor marker. ${ }^{5}$ Polyamines are polyvalent cations, and affect protein synthesis and nucleic acid synthesis by interacting with nucleic acid or other anions. ${ }^{6,7}$ The physiological function of polyamines is not totally understood; however, polyamines are abundant and metabolically active in actively proliferating tissue..$^{5}$ Thus, polyamines might have important roles in the cellular proliferative pathway. A significant elevation of urine diacetylated spermine was reported in urogenital cancer patients. ${ }^{8}$ Furthermore, urine diacetylated spermine concentration was maintained in the low range during the remission period in human cancer patients; however, elevated levels were detected at the reoccurrence period. Therefore, diacetylated spermine might be useful for monitoring response to treatment, and/or reoccurrence. 5

In the veterinary field, although some researchers have investigated the association with other tumor markers, ${ }^{1}$ urine spermidine and spermine, both polyamines, were elevated in dogs with mammary gland tumors. ${ }^{2}$ Consequently, many investigators suggest that diacetylated spermine might be useful as a nonspecific tumor marker and/or early detection marker of tumor reoccurrence, because of the elevations in human cancer patients. The purpose of this study was to evaluate the usefulness of diacetylated spermine as a non-specific tumor marker in dogs.

\section{Materials and Methods}

\section{Animals}

In the present study, 106 dogs were randomly selected and investigated in clinical practice. Urine concentration of diacetylated spermine might be elevated in growing and non-cancer patients (such as those with inflammatory disease). For this reason, animals were divided into 4 groups as follows: cancer-bearing group $(n=23)$, control group $(n=44)$, young group $(\mathrm{n}=24)$ and inflammatory diseases group $(n=15)$. Clinical diagnosis of cancer bearing group and inflammatory diseases group are shown in Tables 1 and 2, respectively. The characteristics of each group are summarized in Table 3.

\section{Sample collection}

Urine samples were collected by cystocente-
Correspondence: Prof. Isaka Mitsuhiro, Marble Veterinary Medical Center, 4-1-6 Ishikawa, Fujisawa, Kanagawa 252-0815, Japan.

Tel. +81.466 .862080 - Fax: +81.466 .860558 .

E-mail: isaka_m@hotmail.com

Key words: diacetylated spermine, urine, tumor marker, canine.

Conflict of interest: the authors report no conflicts of interest.

Received for publication: 10 October 2011. Accepted for publication: 30 November 2011.

This work is licensed under a Creative Commons Attribution NonCommercial 3.0 License (CC BYNC 3.0).

(C) Copyright I. Mitsuhiro et al., 2011

Licensee PAGEPress, Italy

Veterinary Science Development 2011; 1:e16

doi:10.4081/vsd.2011.e16

sis or voiding. For cancer-bearing group, urine samples were collected before treatment and/or during the period of no response to treatment. After screening test, all samples were cryopreserved $\left(-20^{\circ} \mathrm{C}\right)$ for a week.

\section{Measurement}

\section{Screening test}

All collected urine samples underwent sediment and evaluated for WBC, nitrite, urobilinogen, protein, $\mathrm{pH}$, occult blood, specific gravity, keton bodies, bilirubin and glucose (CLINITEL Status ${ }^{\circledR}$ Analyzer; Siemens Co., Ltd., Shinagawa, Tokyo, Japan).

\section{Inflammatory diseases}

Inflammatory diseases were based on the elevation of C-reactive protein (CRP, Laser CRP-2; Arrows Co., Ltd., Ibaraki, Osaka, Japan) and/or leukocyte count (XT-1800i; Sysmex Co., Ltd., Kobe, Hyogo, Japan).

Urine diacetylated spermine and urine creatinine concentration

Urine samples were performed by enzymelinked immunoassay (Metaboscreen Co., Ltd., Yokohama, Kanagawa, Japan) with a commercially manufactured antibody (Mikuri immuno Laboratory Inc., Tanabe, Kyoto, Japan). Urine creatinine concentrations were measured using a Dimension X-pand Plus (Dade Behring Co., Ltd., Shinagawa, Tokyo, Japan). Because of physiological factors and/or circumstances, urine diacetylated spermine concentrations were modified by urine creatinine concentration as followed:

\section{$\frac{\text { [Diacetylated spermine U] }}{\text { [Creatinine U] }} \times 100$}

Several urine samples including diacetylat- 
ed spermine were prepared from cancer dogs to validate our ELISA system; these samples were also evaluated by the commercial available ELISA system (Yamasa 80015; Yamasa

Table 1. Diagnosed by histopathological examination and/or computed tomography.

\begin{tabular}{ll} 
Cancer-bearing group $(\mathrm{n}=23)$ & N \\
Lymphosarcoma & 3 \\
Malignant mammary gland & 5 \\
\hline Mast cell tumor & 1 \\
Malignant melanoma & 1 \\
\hline Hepatocarcinoma & 1 \\
Chronic lymphocytic leukemia & 1 \\
\hline Malignant nerve sheath tumor & 3 \\
Osteosarcoma & 1 \\
\hline Transitional cell carcinoma & 1 \\
Malignant mesenchymal sarcoma & 1 \\
\hline Brain neoplasia & 1 \\
Oral neoplasia & 2 \\
\hline Unknown malignant tumor & 2 \\
\hline
\end{tabular}

Table 2. Diagnosed by clinical examination and laboratory analysis.

\begin{tabular}{ll} 
& N \\
Severe external otitis & 7 \\
Arthritis & 4 \\
\hline Cystitis & 3 \\
Gingivitis & 1 \\
\hline
\end{tabular}

Table 3. Averaged age.

\begin{tabular}{lccr} 
& N & $\begin{array}{c}\text { Sex ratio } \\
\left(\mathrm{M}: \mathrm{F}^{\prime}\right)\end{array}$ & \multicolumn{1}{c}{ Age } \\
Control & 44 & $22: 22$ & $6.95 \pm 0.52$ \\
Inflammation & 15 & $6: 9$ & $5.73 \pm 0.90$ \\
\hline Young & 24 & $12: 12$ & $1.44 \pm 0.14$ \\
Cancer & 23 & $8: 15$ & $10.65 \pm 0.49$
\end{tabular}

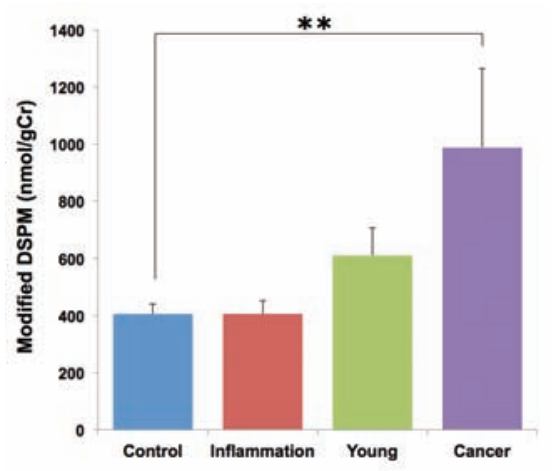

Figure 1. Urine diacetylated spermine concentration in each group (control vs young, $\left.{ }^{* *} \mathrm{P}<0.05\right)$.
Corporation, Choshi, Chiba, Japan). These two systems did not differ significantly in sensitivity and accuracy within 1 to $64 \mathrm{nM}$ diacetylated spermine. Blood contamination of urine samples acquired using general clinical methods does not affect these systems, and the urine was not treated to remove any blood components in this study.

\section{Statistical analysis}

Statistical analysis was performed using commercial statistical software (Stat view 4.0). Statistical analyses were performed with ANOVA with Bonferroni test. A P value $<0.05$ was considered a significant difference.

\section{Results}

There was a significant elevation in the urine diacetylated spermine concentration in the cancer-bearing group, compared to that in the control group $(\mathrm{P}=0.0081)$. Further, the urine diacetylated spermine concentration in the cancer-bearing group did not differ markedly from that in the young group $(\mathrm{P}=0.24)$. However, there was statistic significant different between control and young group dogs $(\mathrm{P}=0.019)$ and between the inflammatory and cancer-bearing groups $(\mathrm{P}=0.62)$.

\section{Discussion}

In this study, the urine diacetylated spermine concentration of the tumor-bearing group only showed a significant elevation compared to the control group. In general, polyamine concentrations are dependent on age, ${ }^{9}$ because they are present in all prokaryotic and eukaryotic cells associated with growth processes. ${ }^{10}$ However, there was no significant elevation in young dogs the compared to control group, although the concentration tended to increase as shown in Figure 1. This phenomenon might be associated with systemic cellular proliferation. Taken together, although we did not evaluate the age-dependent elevation of urine diacetylated spermine, we suggest that diacetylated spermine is not useful as a tumor marker in dogs under the age of 2 years. It was reported that the urine diacetylated spermine concentration was elevated in acute appendicitis, cholecystitis, inflammatory bowel disease, viral load, steroids, and low calorie diet.11,12 However, there was no significant elevation in inflammation compared to the control group in our study. To the best of our knowledge, there was no previous report on the association among CRP, WBC count, and diacetylated spermine. Thus, the dissociation recognized in our study could not be com- pletely explained.

On the other hand, urine diacetylated spermine was significantly elevated in patients with cancer. ${ }^{3}$ In the present study, we found a significant elevation in the cancer-bearing group. We selected malignant tumors for the cancer-bearing group, because in the comparison study between malignant and benign bladder tumors in humans. ${ }^{8}$ Thus, it was suggested that the concentration of urine diacetylated spermine might depend on tumor type and malignancy/benignity. Although we did not evaluate these parameters in the present study, veterinarians should consider the sensitivity and specificity of the examination method. These are statistical measures of the performance of a binary classification test. Sensitivity measures the proportion of actual positives that are correctly identified as such. Specificity measures the proportion of negatives that are correctly identified. In the near future, we shall perform further study to elucidate these parameters of urine diacetylated spermine concentration. However, a polyamine biosynthesis inhibitor has been used for human cancer patients in a phase I study; 13 thus, the diverse research on polyamines will lead to the development of new anti-cancer drugs and non-specific tumor markers in the future.

\section{Conclusions}

In our study, the urine diacetylated spermine concentration was significantly elevated in cancer-bearing dogs, and this may be clinically useful in confirming the presence of canine tumors to the owners. However, further study in the near future is warranted.

\section{References}

1. Allen DK, Waters DJ, Knapp DW, Kuczek T. High urine concentrations of basic fibroblast growth factor in dogs with bladder cancer. J Vet Intern Med 1996;10:231-4.

2. Motyl T, Ratajska K, Jurga W, et al. Polyamines and pyrimidines in blood plasma and urine of dogs bearing mammary tumours. Zentralbl Veterinarmed A 1994;41:766-9.

3. Russell DH, Levy CC, Schimpff SC, Hawk IA. Urinary polyamines in cancer patients. Cancer Res 1971;31:1555-8.

4. Pegg AE. Recent advances in the biochemistry of polyamines in eukaryotes. Biochem J 1986;234:249-62.

5. Kawakita M, Hiramatsu K. Diacetylated derivatives of spermine and spermidine as novel promising tumor markers. J 
Biochem 2006;139:315-22.

6. Burton DR, Forsén S, Reimarsson P. The interaction of polyamines with DNA: a23Na NMR study. Nucleic Acids Res 1981;9:1219-28.

7. Balasundaram D, Tyagi AK. Polyamine-DNA nexus: structural ramifications and biological implications. Mol Cell Biochem 1991;100:129-40.

8. Stejskal D, Humenanska V, Hanulova Z, et al. Evaluation of urine N1, N12Diacetylspermine as potential tumor marker for urinary bladder cancer. Biomed Pap Med Fac Univ Palacky Olomouc Czech
Repub 2006;150:235-7.

9. Casti A, Orlandini G, Reali N, et al. Pattern of blood polyamines in healthy subjects from infancy to the adult age. J Endocrinol Invest 1982;5:263-6.

10. Bachrach U, Wang YC, Tabib A. Polyamines: new cues in cellular signal transduction. News Physiol Sci 2001;16: 106-9.

11. Enjoji M, Kotoh K, Nakamuta M. Factors affecting the increase of urinary diacetylspermine levels: a study in patients with liver diseases. Rinsho Byori 2006;54:126-31.
12. Hiramatsu K, Takahashi K, Yamaguchi T, et al. N(1),N(12)-Diacetylspermine as a sensitive and specific novel marker for early- and late-stage colorectal and breast cancers. Clin Cancer Res 2005;11:2986-90.

13. Siu LL, Rowinsky EK, Hammond LA, et al. A phase I and pharmacokinetic study of SAM486A, a novel polyamine biosynthesis inhibitor, administered on a daily-timesfive every-three-week schedule in patients with advanced solid malignancies. Clin Cancer Res 2002;8:2157-66. 